\title{
Anhedonia in chronic pain and prescription opioid misuse
}

\author{
Eric L Garland, \\ University of Utah
}

\author{
Martin Trøstheim, Marie Eikemo, \\ Gernot Ernst, Siri Leknes \\ University of Oslo
}

\begin{abstract}
BACKGROUND: Both acute and chronic pain can disrupt reward processing. Moreover, prolonged prescription opioid use and depressed mood are common in chronic pain samples. Despite the prevalence of these risk factors for anhedonia, little is known about anhedonia in chronic pain populations.
\end{abstract}

METHODS: We conducted a large-scale, systematic study of anhedonia in chronic pain, focusing on its relationship with opioid use/misuse, pain severity, and depression. Chronic pain patients across four distinct samples $(\mathrm{N}=488)$ completed the Snaith-Hamilton Pleasure Scale (SHAPS), measures of opioid use, pain severity and depression, as well as the Current Opioid Misuse Measure (COMM). We used a meta-analytic approach to determine reference levels of anhedonia in healthy samples spanning a variety of countries and diverse age groups, extracting SHAPS scores from 58 published studies totaling 2664 psychiatrically healthy participants.

RESULTS: Compared to healthy samples, chronic pain patients showed higher levels of anhedonia, with $\sim 25 \%$ of patients scoring above the standard anhedonia cut-off. This difference was not primarily driven by depression levels, which explained less than $25 \%$ of variance in anhedonia scores. Neither opioid use duration, dose, nor pain severity alone was significantly associated with anhedonia. Yet, there was a clear effect of opioid misuse, with opioid misusers $(C O M M \geqslant 13)$ reporting greater anhedonia than non-misusers. Opioid misuse remained a significant predictor of anhedonia even after controlling for pain severity, depression and opioid dose.

CONCLUSIONS: Study results suggest that both chronic pain and opioid misuse contribute to anhedonia, which may, in turn, drive further pain and misuse.

Keywords: addiction; depression; hedonic; opioid; positive affect; reward

Does being in chronic pain preclude the enjoyment of rewards? There are several routes through which pain could inhibit reward processing (Schwartz et al., 2014). For example, pain's attention-grabbing quality

Corresponding author: Eric L. Garland, 395 South, 1500 East, University of Utah, Salt Lake City, UT 84112, elgar$\underline{\text { lan@gmail.com }}$

Acknowledgements: This work was supported by supported by R01DA042033, R34DA037005, and R03DA032517 (PI: Garland) from the National Institute on Drug Abuse. The content is solely the responsibility of the authors and does not necessarily represent the official views of the National Institutes of Health. The authors declare no conflicts of interest with respect to the research contained herein. We are thankful to Kongsberg hospital's orthopedic ward nurses for data collection. could distract from rewards (Eccleston and Crombez, 1999). Also, the stress caused by enduring pain could inhibit reward processing (Porcelli and Delgado, 2017). The high comorbidity between chronic pain and depression could be another cause. Anhedonia, defined as the impaired capacity to experience pleasure from naturally rewarding objects and events, is one key symptom of depression.

Surprisingly little data on anhedonia in chronic pain is available in the literature. Nearly four decades ago, Marbach and colleagues (Marbach and Lund, 1981, Marbach et al., 1983) found significantly more physical anhedonia in arthritic but not facial pain patients. Depression scores showed only a modest relationship with anhedonia across arthritic and facial pain patients. 
Two recent studies of chronic pain reported elevated scores on anhedonia-related items of depression questionnaires (Elvemo et al., 2015), which were partly related to the presence of breakthrough pain (Narayana et al., 2015). However, a new study using a validated anhedonia questionnaire found that only a small proportion of abdominal pain patients exhibited anhedonia above the validated cutoff (Carpinelli et al., 2019). In summary, the extant literature, though limited, suggests that depression may not be the primary mechanism linking chronic pain to anhedonia.

Another potential route through which chronic pain could cause anhedonia is via disrupted opioidergic signaling in the brain. Several molecular imaging studies indicate alterations in endogenous opioid tone in chronic pain samples (Harris et al., 2007, Martikainen et al., 2013). These changes are believed to result from pain-induced reductions in mu-opioid receptor expression and have been linked to anhedonia (2018). Mu-opioid receptor signaling in humans is thought to downregulate pain (Sprenger et al., 2006, Zubieta et al., 2001) and upregulate pleasure (Buchel et al., 2018, Chelnokova et al., 2014, Eikemo et al., 2016, Price et al., 2016). Moreover, many patients receive prescription opioid treatment for chronic pain. Indeed, extended opioid therapy is theorized to cause anhedonia via dopaminergic and opioidergic mechanisms integral to hedonic function (Volkow and McLellan, 2016).

Furthermore, misuse of opioids occurs with some frequency in opioid-treated chronic pain patients (Vowles et al., 2015). Prescription opioid misuse is theorized to further exacerbate hedonic deficits in chronic pain populations (Garland et al., 2013), consistent with the role of anhedonia in other substance dependence (Franken et al., 2007, Garfield et al., 2017, Huhn et al., 2016b, Stevens et al., 2007). According to the allostatic model (Koob and Le Moal, 2001, Koob and Moal, 1997), opioid misuse causes neuroadaptations in cortico-limbic-striatal stress and reward systems in the brain, resulting in hedonic dysregulation. In support of this notion, opioid misusing chronic pain patients showed significantly reduced autonomic and attentional responses to naturally rewarding stimuli relative to medicationadherent chronic pain patients (Garland et al., 2017, Garland et al., 2015a). However, whether opioid misusing chronic pain patients report reduced ability to experience pleasure from everyday rewards has yet to be determined.

Indeed, the field lacks up-to-date knowledge on the extent of anhedonia in chronic pain populations. Here, we administered a frequently used anhedonia questionnaire (Snaith et al., 1995) in four separate samples of chronic pain patients (total $\mathrm{N}=488$ ), and compared anhedonia scores in these samples to a metaanalytically derived reference value from 2664 healthy controls. Further, participants in each of the three opioid-treated chronic pain samples were classified as opioid misusers or non-misusers (i.e., medication adherent) according to a validated cut-point for opioid misuse. We hypothesized that chronic pain patients would display greater anhedonia than psychiatrically healthy controls, and that patients who misused opioids would have more severe anhedonia. We further hypothesized that anhedonia in these chronic pain samples would be partially independent of depression scores.

\section{Methods}

\subsection{Overview of data collection}

We administered the Snaith-Hamilton Pleasure Scale (SHAPS) (Snaith et al., 1995) and the Current Opioid Misuse Measure (COMM) (Butler et al., 2007) to independent samples of chronic pain patients. Data were collected in three separate research projects assessing emotional and cognitive factors implicated in opioid misuse among individuals with chronic pain who had received prescription opioid analgesics for $>90$ days, and in a fourth project assessing anhedonia in non-opioid treated chronic pain patients. Sample one consisted of civilian patients $(\mathrm{N}=115)$ recruited between 2011-2012 from primary care and pain clinics in the Southeastern U.S. Sample two consisted of military personnel $(\mathrm{N}=35)$ recruited between 20132015 via provider referral from primary care, an interdisciplinary pain program, and a substance abuse program on an Army base in the U.S. Intermountain West. Sample three consisted of civilian patients $(\mathrm{N}=281)$ recruited between 2015-2018 from primary care and pain clinics in the U.S. Intermountain West. Data for a fourth sample $(\mathrm{N}=56)$ referred for hip or knee-joint replacement was collected in 2019 at a Norwegian hospital. These patients reported comparable chronic pain intensity to the opioid-treated samples but were not treated with opioid analgesics (seven patients reported intermittent codeine intake). 


\subsection{Participants}

For the chronic pain samples, inclusion criteria were: being at least 18 years old; having chronic noncancer-related pain (self-reported and confirmed through medical chart review or clinical interview). Opioid-treated samples were additionally required to have used prescription opioid analgesics for $\geq$ five days a week for the past 90 days or more (Chou et al., 2009). Daily use of opioids was an exclusion criterion for the sample of non-opioid-using patients. Participants were excluded if they were actively suicidal or psychotic according to the Mini-International Neuropsychiatric Interview 6.0 (MINI) (Sheehan et al., 1998).

\subsection{Measures}

Anhedonia. The SHAPS consists of 14 items tapping the pleasure experienced from a variety of natural rewards (e.g., being with family, a warm bath, smiling faces, a beautiful landscape, receiving praise), rated on a Likert-type scale (1=strongly agree, $4=$ strongly disagree). Using this scoring rubric, SHAPS total scores can range from 14 to 56, with higher scores indicating higher levels of anhedonia (Franken et al., 2007, Snaith et al., 1995). Internal reliability across all four samples was adequate, with alpha coefficients from .78. to .92. To determine the proportion of each sample exceeding Snaith's suggested cutoff for clinical anhedonia (disagreeing with three or more out of the 14 items) and to facilitate comparison with data analyzed other scoring methods, we also used the 0-1 scoring system employed by Snaith et al. (1995). The original and Norwegian (Eikemo et al., 2016) versions of the SHAPS were used.

Pain. Pain was measured using the original and Norwegian (Klepstad et al., 2002) versions of the Brief Pain Inventory.

Opioid misuse. On the COMM (Butler et al., 2007), opioid-treated participants responded to 17 items rated on a Likert-type scale $(0=$ never, $4=$ very often) regarding how often in the past 30 days they had engaged in behaviors linked with opioid misuse (e.g., took opioid medication in excessive doses, took medication in ways other than how it was prescribed). Internal reliability across all three samples was adequate, with alpha coefficients from .79 to .85. A study of a broad sample of opioid-treated chronic pain patients found via receiver-operator characteristic curve analyses that a score of 13 or higher on the COMM had maximum sensitivity and specificity to identify high risk for opioid misuse consistent with opioid use disorder (Meltzer et al., 2011). We used this COMM threshold value to minimize false positives and define groups because our recruited samples were similar to those of Meltzer et al. (2011).

Opioid dose and duration of opioid use were obtained via self-report and corroborated by medical chart review. In sample 4, opioid use was additionally cross-checked by the Norwegian Prescription Database, where prescription drugs of every Norwegian patient are documented. Opioid doses were converted to morphine milligram equivalents using equianalgesic dose ratios established by guidelines from the Centers for Disease Control (CDC).

Presence of major depressive disorder and major depressive disorder severity (total depression symptom count for current and past episodes) were established during psychiatric screening by trained clinical staff (e.g., psychologists, social workers, nurses) via the Structured Clinical Interview for DSM (SCID; sample 2) and MINI (samples 1 and 3). Beck's Depression Inventory (BDI) scores were collected from sample 4; scores were supplemented by a clinical interview.

The University of Utah institutional review board (IRB) approved data collection for samples 2 and 3. Florida State University IRB approved data collection for sample 1, whereas data collection for sample 4 was approved by the Regional Ethics Committee (2018/1016 REK Sør-Øst) of Norway.

\subsection{Data Analysis \\ 2.4.1 Meta-analysis}

The first aim of our meta-analysis was to establish a reference value on the SHAPS (a general mean and confidence interval) for psychiatric healthy samples based on the existing literature, and to compare this value to that of the chronic pain samples. A second objective was to compare the anhedonia symptoms across the opioid-treated patient subgroups with and without symptoms of opioid misuse. Studies citing the original SHAPS publication (Snaith et al., 1995) were identified with SCOPUS, Web of Science and PubMed through April 2018. We included studies in all languages that:

1) Included original data

2) Used the complete SHAPS with four-point scoring of items

3) Included at least one sample of participants described as having no current or recent psychiatric conditions.

4) Assessed SHAPS at baseline or in a notreatment condition 
5) Did not perform selective recruitment of participants based on SHAPS score

6) Reported SHAPS data from analyses performed without adjusting for covariates.

Data was extracted from 58 studies. When necessary, we e-mailed corresponding authors $(\mathrm{k}=36)$ to obtain missing data. To enable comparison, SHAPS scores from studies using 0-3, 4-1 or other variants of 4-point scoring of the SHAPS were recalculated to conform to a 1-4 scoring method in which 1 represents 'strongly agree' and 4 represents 'strongly disagree' (Franken et al., 2007). Descriptive statistics from these studies were entered in a meta-analysis together with the data from the patient samples.

For the meta-analysis of SHAPS scores, we used random-effects models implemented in the "metafor" package (Viechtbauer, 2010) in R statistical software (R Core Team, 2018). Random-effects models were chosen due to the assumed heterogeneity in SHAPS scores across patient and healthy samples. Sample means, standard deviations and number of participants in each sample were used as input data. We computed separate random-effects models for the healthy samples and for each of the patient samples. We also computed random-effects models for the subsamples of pain patients whose COMM scores indicated the presence of absence of opioid misuse. The DerSimonian-Laird (1986) method was used to estimate the between-studies variance for each randomeffects model.

Confidence intervals (CI; 95\%) for the summary effects were calculated using critical zvalues. We calculated a 95\% prediction interval (PI) for the summary effect of each group using a bootstrapping procedure introduced by Nagashima et al. (2018) and implemented in the "pimeta" package in R. The PI accounts for heterogeneity and predicts the true effect of a new study given past studies. This method for calculating PI has good coverage probability even when the number of studies is small. 100000 bootstrap samples were used to estimate the $95 \%$ prediction interval for each summary effect.

\subsubsection{Comparisons of groups}

We compared the summary SHAPS scores of the different groups and subgroups using $\mathrm{Z}$ tests.

\subsubsection{Control analysis}

To control for any differences in age and percentage of women between healthy and patient samples, we performed a meta-regression using the "metafor" package and used Z-tests to test for significant group differences.

\subsubsection{Analysis of individual SHAPS items}

To address the question of whether anhedonia in chronic pain is driven by a specific subset of everyday rewards, we calculated mean scores and 95\% CIs for each of the 14 SHAPS items across the four patient samples using random-effects models. The DerSimonian-Laird (1986) method was used to estimate the between-studies variance.

\subsubsection{Analysis of variance within the chronic pain samples}

We computed zero-order correlations between primary study variables (pain severity, depression, opioid dose and duration of opioid medication) across all opioid-treated samples. To assess the extent to which the association between opioid misuse (dichotomous: misuser yes/no) and SHAPS scores were independent of these variables, we ran a series of mixed models to control for clustering by sample (via SPSS 22.0). Sample number was specified as a clustering variable by including a random intercept for sample number. Including random slopes resulted in lack of model convergence due to random slope variance estimates being zero. Hence, following a forward stepping model building approach (Raudenbush and Bryk, 2002; Snijders and Boskers, 1999), variables with zero estimates were removed as random slope effects from the final model, but were retained as fixed effects. Thus, the equation for the final model with random intercept and fixed slopes is as follows:

Level 1

$$
\begin{aligned}
& \mathrm{yij}=\beta 00+\beta 10 \mathrm{Xij}+\beta 20 \mathrm{Xij}+\beta 30 \mathrm{Xij}+\beta 40 \mathrm{Xij} \\
& +\varepsilon \mathrm{ij} \\
& \mathrm{yij}=\beta 00+\beta 10(\text { opioid misuse }) \mathrm{ij}+\beta 20(\text { depression } \\
& \text { symptom severity)ij }+\beta 30(\text { opioid dose }) \mathrm{ij}+ \\
& \beta 40(\text { pain severity)ij }+\varepsilon \mathrm{ij}
\end{aligned}
$$

Level 2

$$
\begin{aligned}
& \beta 0 \mathrm{j}=\Upsilon 00+u 0 j \\
& \beta 1 \mathrm{j}=\Upsilon 10 \\
& \beta 2 \mathrm{j}=\Upsilon 20 \\
& \beta 3 \mathrm{j}=\Upsilon 30 \\
& \beta 4 \mathrm{j}=\Upsilon 40
\end{aligned}
$$

We then conducted a sensitivity analysis in which opioid use duration and depression symptom 
Table 1. Demographic and clinical characteristics

\begin{tabular}{|c|c|c|c|c|}
\hline Measure & $\begin{array}{l}\text { Sample } 1 \\
N=115\end{array}$ & $\begin{array}{c}\text { Sample } 2 \\
N=35\end{array}$ & $\begin{array}{c}\text { Sample } 3 \\
N=282\end{array}$ & $\begin{array}{c}\text { Sample } 4 \\
N=56\end{array}$ \\
\hline Age, $M \pm$ s.D. & $48.3 \pm 13.6$ & $32.9 \pm 8.4$ & $52 \pm 12.5$ & $67.8 \pm 9.7$ \\
\hline Female, $N(\%)$ & $78(68 \%)$ & $3(8.6 \%)$ & $178(63.3 \%)$ & $32(57 \%)$ \\
\hline \multicolumn{5}{|l|}{ Primary pain condition, $N(\%)$} \\
\hline Low back & $65(56.5 \%)$ & $24(68.6 \%)$ & $147(52.3 \%)$ & $0(0 \%)$ \\
\hline Joint/extremity & $8(7.0 \%)$ & $5(14.3 \%)$ & $43(15.3 \%)$ & $56(100 \%)$ \\
\hline Fibromyalgia & $23(20.0 \%)$ & $0(0 \%)$ & $31(11.0 \%)$ & $0(0 \%)$ \\
\hline Neck/shoulder & $7(6.1 \%)$ & $4(11.4 \%)$ & $23(8.2 \%)$ & $0(0 \%)$ \\
\hline Neuropathic/neurological & $4(3.5 \%)$ & $0(0 \%)$ & $22(7.8 \%)$ & $0(0 \%)$ \\
\hline Other & $8(7.0 \%)$ & $2(5.7 \%)$ & $15(5.3 \%)$ & $0(0 \%)$ \\
\hline Pain severity (BPI, $0-10), M \pm$ s.D. & $5.5 \pm 1.5$ & $5.6 \pm 1.8$ & $5.4 \pm 1.5$ & $5.1 \pm 1.9^{\mathrm{a}}$ \\
\hline Morphine equivalent daily dose, $M \pm$ S.D. & $180.2 \pm 469.9^{b}$ & $44.2 \pm 18.2$ & $98.8 \pm 242.8^{c}$ & 0 \\
\hline Duration of opioid use (years), $M \pm$ S.D. & $8.8 \pm 10.0^{d}$ & - & $9.2 \pm 7.8^{\mathrm{e}}$ & 0 \\
\hline Opioid misuse score (COMM), $M \pm$ S.D. & $17.9 \pm 9.7$ & $11.4 \pm 6.9$ & $15.75 \pm 9.0$ & - \\
\hline Opioid misusers, $N(\%)$ & $83(72.2 \%)$ & $14(40.0 \%)$ & $159(56.6 \%)$ & $0(0 \%)$ \\
\hline Depression $^{f}$ & $69(68.3 \%)$ & $9(25.7 \%)$ & $100(36.1 \%)$ & $9(16.1 \%)$ \\
\hline Anhedonia score (SHAPS, $1-4$ ), $M \pm$ S.D. & $24.4 \pm 6.7$ & $25.1 \pm 7.7$ & $24.6 \pm 7.3$ & $23.9 \pm 5.8$ \\
\hline Anhedonia score (SHAPS, $0-1$ ) $M \pm$ S.D. & $1.6 \pm 2.6$ & $2.3 \pm 2.7$ & $1.8 \pm 2.6$ & $1.3 \pm 1.9$ \\
\hline Anhedonic, $N(\%)$ & $22(19.1 \%)$ & $12(34.3 \%)$ & $80(28.5 \%)$ & $8(14 \%)$ \\
\hline
\end{tabular}

- = data missing;

${ }^{a}=$ due to missing data, $n=55$;

$\mathrm{b}=$ due to missing data, $n=69$;

${ }^{c}=$ due to missing data, $n=269$;

$\mathrm{d}=$ due to missing data, $n=74$;

${ }^{\mathrm{e}}=$ due to missing data, $n=227$;

$\mathrm{f}=N(\%)$ major depressive disorder (sample 1-3); Beck depression inventory $>13$ (sample 4).

Table 2. Characteristics of healthy and patient groups

\begin{tabular}{lllll}
\hline & Healthy & Chronic pain (total) & Nonusers & Misusers \\
\hline$k$ & 58 & 4 & 1 & 3 \\
\hline$N$ & 2664 & 488 & 56 & 3 \\
\hline$N$ female (\%) & $1484(56 \%)$ & $291(60 \%)$ & $32(57 \%)$ & 176 \\
\hline Mean age (s.o.) & $31.40(9.29)$ & $51.97(12.10)$ & $67.75(9.71)$ & $52.57(12.72)$ \\
\hline Range & $13.04-70.60$ & $32.88-67.75$ & - & $38.10(12.15)$ \\
\hline
\end{tabular}

${ }^{\mathrm{a}}$ Mean weighted by individual study Ns, pooled S.D..

count were included in the model as covariates. We also examined the covariance between SHAPS scores and opioid misuse as a continuous variable (total COMM score).

\section{Results}

A total of 488 chronic pain patients were included across the four study samples (Table 1 ). The majority of patients were Caucasian; $60 \%$ (291) of patients were women. Across the three opioid-treated samples, the most commonly reported primary pain condition was low back pain (54.7\%), followed by joint/extremity pain (12.9\%), fibromyalgia pain (12.5\%), neck/shoulder pain (10.2\%), neuropathic/neurological (6.0\%), and other $(5.8 \%)$. The mean pain severity was $5.48(S D=$ 1.51) out of 10 , for which patients had taken opioids for an average of $9.10(S D=8.34)$ years. The average morphine equivalent daily dose was $100.14(S D=242.89)$ $\mathrm{mg}$. The fourth, non-opioid treated sample consisted of patients referred for hip or knee replacement with mean pain severity of $5.1 \pm 1.9$. Approximately one in four of the chronic pain participants included were anhedonic according to Snaith et al.'s suggested cutoff for clinical anhedonia (reporting no projected enjoyment of three or more of the 14 items; Sample 1: 19.1\%; Sample 2: 
34.3\%; Sample 3: 28.5\%, Sample 4: 14\%) (Snaith et al., 1995).

The 58 healthy samples included in the metaanalysis consisted of 2664 participants, 1484 (56\%) of whom were women (Table 2). The mean ages of the healthy samples ranged from 13.04 to 70.60 with a weighted mean age of $31.4(S D=9.3)$. Compared to the healthy samples, the patient samples covered a considerably narrower mean age range (32.9-67.8) and consisted of older participants $(M=52.0, S D=12.1, z=$ $35.0, p<.001$, two-tailed). The proportion of female participants did not differ significantly between the patient group and the healthy group $(z=1.6, p=.11$, twotailed). To exclude any effects of age or gender differences on SHAPS scores, we performed additional meta-regressions to control for mean age and gender proportions.

\subsection{Meta-analyses}

Random-effects models for each group and subgroup are presented in table 3 (see also figure 1 and figure 2). Results from z-tests of difference in meta-analytic means are available in table 4 . Healthy participants yielded an average score of $20.08(S E=0.28)$ on the SHAPS. Pain patients' scores were significantly higher at $24.46(S E=0.32, p<.001$, two-tailed $)$. Opioid-treated, non-misusing pain patients displayed significantly higher anhedonia scores $(M=23.15, S E=$ $0.55)$ than healthy controls $(p<.001$, two-tailed). Patients who misused opioids showed the highest anhedonia scores $(M=26.28, S E=1.12)$, which were significantly higher than those of non-misusers $(p=.01$, two-tailed).

Table 4. Comparisons of SHAPS total scores under separate random-effects models ${ }^{a}$ for each group

\begin{tabular}{llcc}
\hline Comparison & $\begin{array}{c}\text { Difference } \\
(\text { S.E. })^{\mathrm{b}}\end{array}$ & $z$ & $p^{c}$ \\
\hline $\begin{array}{l}\text { Healthy v. chronic pain } \\
\text { (total) }\end{array}$ & $4.37(0.42)$ & 10.33 & $<0.001$ \\
\hline Healthy v. non-misusers & $3.07(0.62)$ & 4.95 & $<0.001$ \\
\hline Healthy v. misusers & $6.20(1.16)$ & 5.36 & $<0.001$ \\
\hline Non-misusers v. misusers & $3.13(1.25)$ & 2.51 & 0.01 \\
\hline
\end{tabular}

${ }^{\mathrm{a} D}$ DerSimonian andLaird (1986) estimator of between-studies variance.

b On the same scale as the SHAPS.

'Two-tailed.

\subsubsection{Prediction intervals}

The range of the $95 \%$ prediction intervals (PIs) for the healthy group was 16.03-24.12, overlapping somewhat with the PI of the opioid-treated chronic pain patients [23.15-25.68]. The non-misuser subgroup's 95\% PI was 20.20-25.70, overlapping substantially with the misuser subgroup's PI whic was 15.31-37.58.

\subsubsection{Control analysis}

Even when adjusting for age and gender, group type remained a significant predictor of SHAPS scores in the meta-regression $\left(B_{\text {Group }}=5.28, S E=1.09, z=\right.$ 4.86, $p<.001 ; B_{\text {Age }}=-0.06, S E=0.02, z=-2.39, p=$ $.02 ; B \%$ female $=-0.02, S E=0.01, z=-1.29, p=.20$ ). This indicates that whereas the chronic pain samples on average have SHAPS scores 5.28 points higher than healthy samples, an increase in average sample age of one year corresponds to a 0.06-point decrease in SHAPS scores, i.e. a negligible effect of age and gender distribution.

\subsubsection{Sensitivity analysis of meta-analysis}

To assess whether the results were dependent on our choice of the DerSimonian-Laird method for estimating the between-studies variance (i.e. $T^{2}$ ), we repeated all the above meta-analyses using other recommended $t^{2}{ }^{2}$ estimators for continuous data, including the restricted maximum likelihood (REML) and PauleMandel (PM) methods (Veroniki et al., 2016). The results from these analyses were fully consistent with those reported above.

\subsection{Analysis of individual SHAPS items}

Mean item-level scores ranged from 1.44 to 2.2 (Supplementary Figure 1 and Supplementary Table 1). This constricted range indicated comparable levels of anhedonia across differing types of rewards (i.e., generalized anhedonia), rather than anhedonia in response to a specific type of reward. 


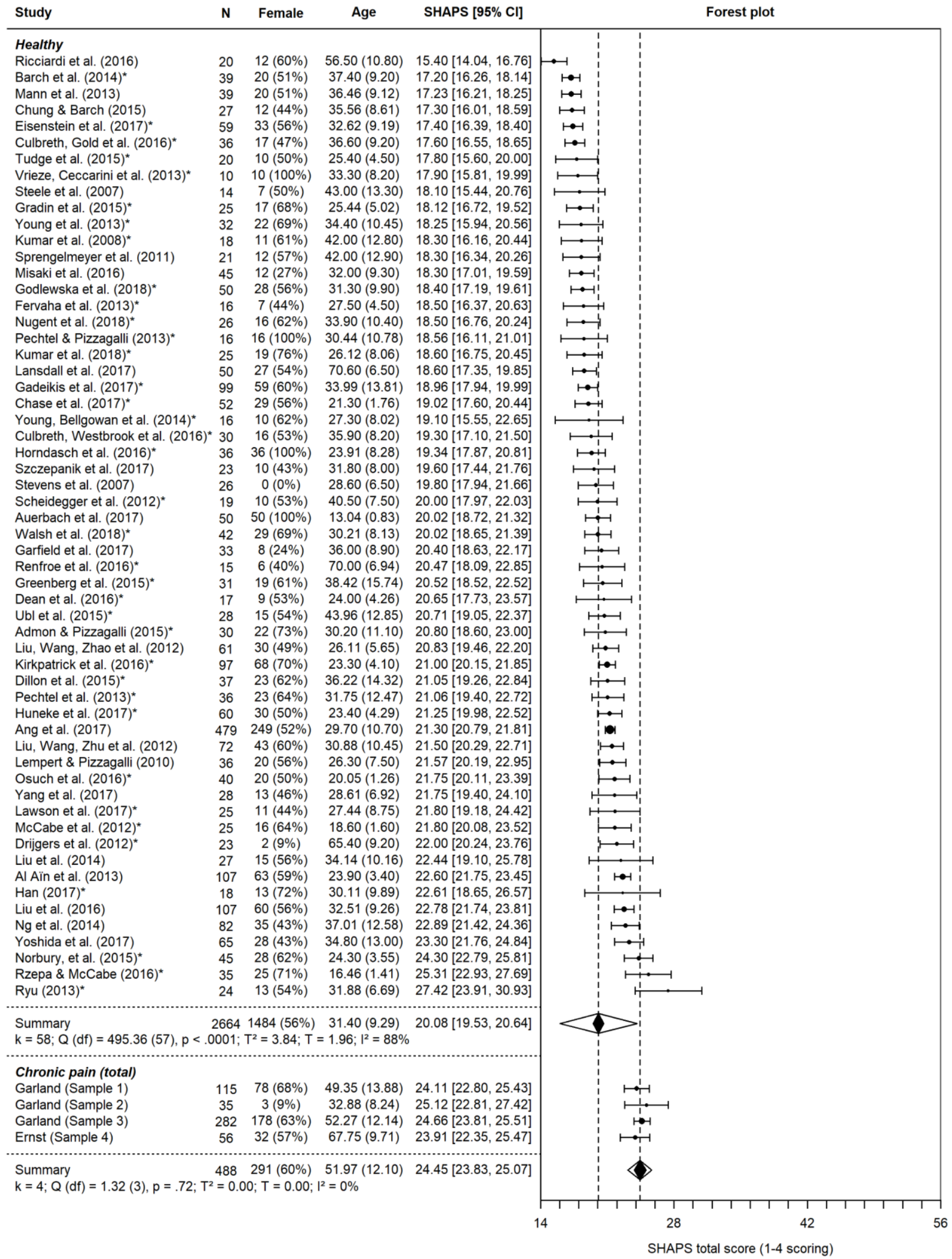

Figure 1. Forest plot of individual study SHAPS scores and summary SHAPS scores based on separate random-effects models for healthy samples and patient samples. The dotted lines indicate the summary SHAPS score of each group. The black polygons indicate the summary SHAPS score and 95\% confidence intervals (Cls) of each group while the transparent overlapping polygons indicate 95\% prediction intervals (PIs) of each group. 95\% Cls were calculated using critical t-values for individual studies and critical z-values for summary effects. The lack of overlap between $\mathrm{Cls}$ for healthy and pain groups indicate significant differences. $\mathrm{T}_{2}$ is the estimate of the between-studies variance based on the DerSimonian and Laird (1986) method. I indicates the percentage of total variation in SHAPS scores across studies that is due to heterogeneity rather than chance (Higgins et al., 2003). Cochran's $Q$ is used to test if there is variation in the observed study effects that cannot be explained by sampling error. Note. *Received missing data. Ricciardi et al. (2016) used the SHAPS-C (modified for clinician administration; Ameliet al., 2014). 


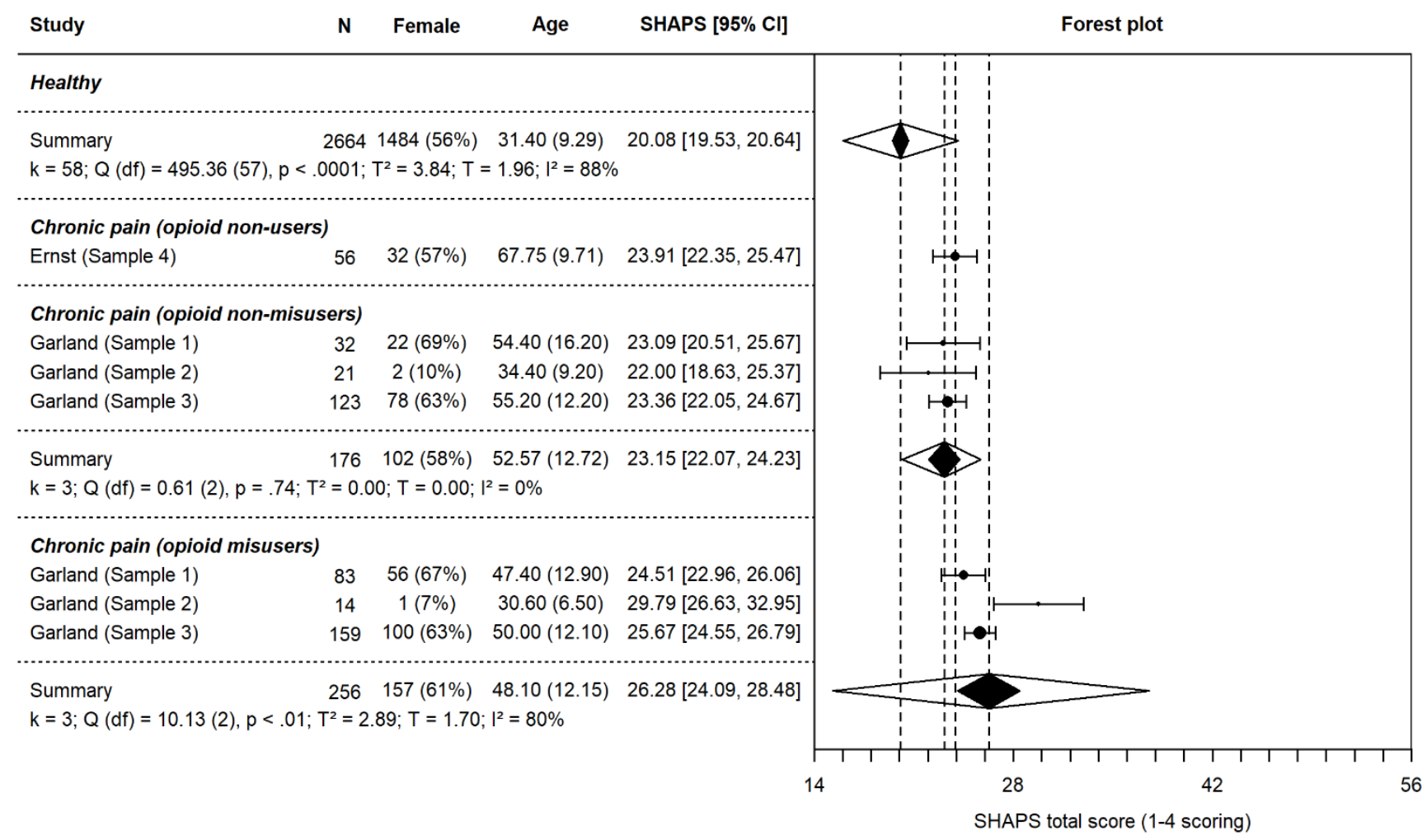

Figure 2. Forest plot of individual study SHAPS scores and summary SHAPS scores based on separate random-effects models for subsamples of chronic pain patients with and without opioid misuse. The summary SHAPS score for healthy samples is the same as in Fig. 1. The dotted lines indicate the summary SHAPS score of each group. The black polygons indicate the summary SHAPS score and $95 \%$ confidence intervals (CIs) of each group while the transparent overlapping polygons indicate $95 \%$ prediction intervals (PIs) of each group. $95 \%$ CIs were calculated using critical t-values for individual studies and critical z-values for summary effects. The lack of overlap between CIs for healthy and pain groups indicate significant differences. T2 is the estimate of the between-studies variance based on the DerSimonian and Laird (1986) method. I 2 indicates the percentage of total variation in SHAPS scores across studies that is due to heterogeneity rather than chance (Higgins et al., 2003). Cochran's $Q$ is used to test if there is variation in the observed study effects that cannot be explained by sampling error.

\subsection{Analysis of variance within chronic pain samples}

\subsubsection{Sample 1-3}

In zero-order correlations, anhedonia scores were positively correlated with depression symptom count $(r=.26, p<.001)$ and shared approximately $7 \%$ of common variance, but were not significantly associated with opioid use duration $(r=.05, p=.44$; Sample 3 only), opioid dose $(r=.09, p=.13)$, or pain severity $(r=.09, p=.08)$.

To assess whether anhedonic symptoms are greater in opioid-treated patients classified as misusers compared to those classified as non-misusers after controlling for individual differences in pain severity, MDD diagnosis, and opioid dose, we computed a mixed model (Supplementary Table 2). As an estimate of clustering by sample study, in the unconditioned model, the ICC was $<.00001$. Although we specified a random intercept for sample number, model convergence criteria were not met because random intercept covariance estimates were zero, indicating that the model was unable to uniquely estimate any variation from sample to sample above and beyond the residual variance from individual to individual. Thus, the random intercept was dropped from the model. In this model (model 1), neither pain severity, MDD diagnosis, nor opioid dose significantly predicted anhedonia, whereas opioid misuse status remained a significant predictor of anhedonia $(B=3.12, S E=0.88, p<.001)$. The final model indicated that after controlling for pain severity, MDD diagnosis, and opioid dose, misusers continued to exhibit significantly higher anhedonia ( $M$ $=26.29, S E=0.56)$ than non-misusers $(M=23.17, S E$ $=0.65)$. 


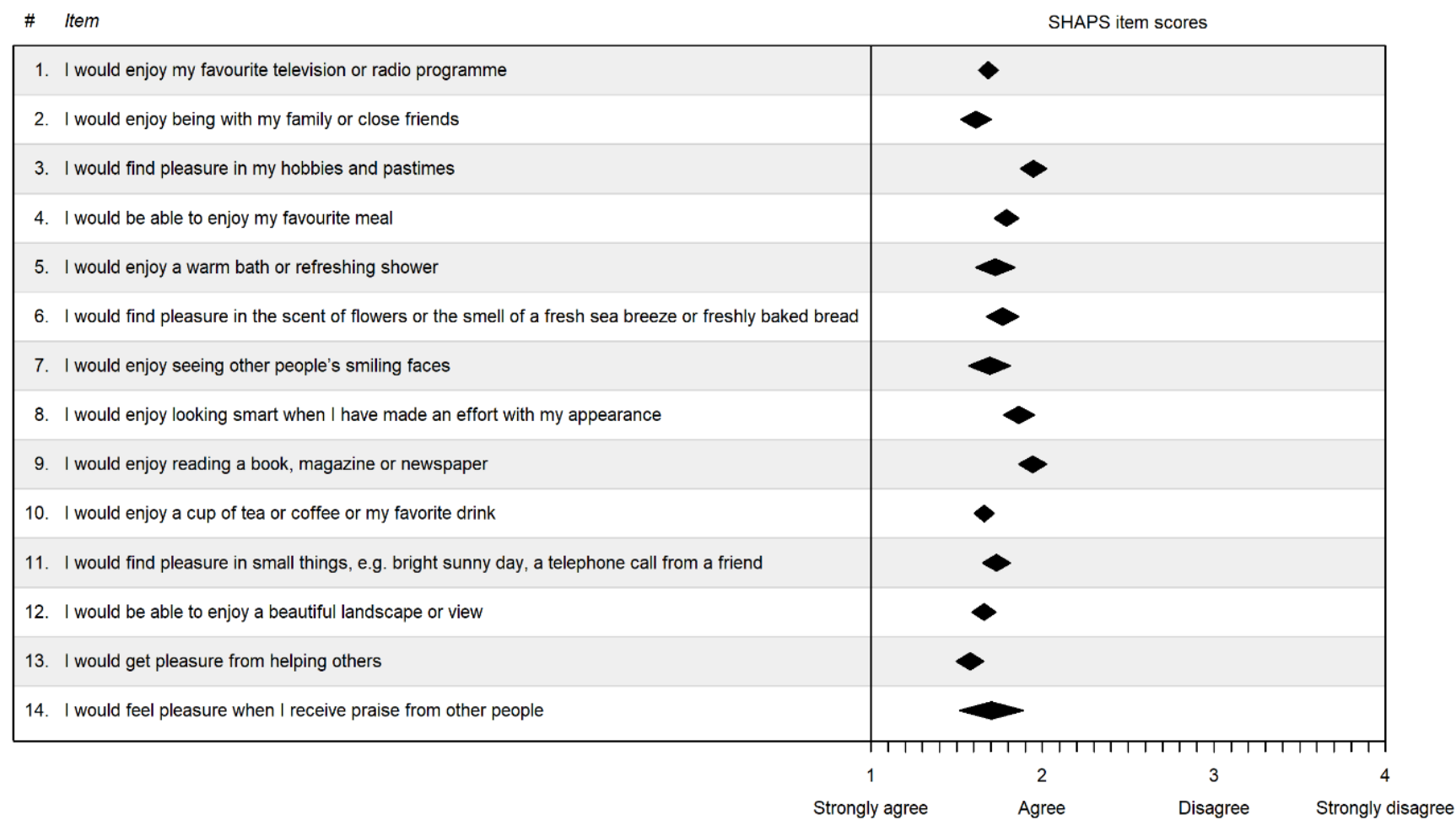

Figure 3. Meta-analysis of item-level SHAPS scores in chronic pain patients showed rather consistent scores across items.

We next computed a mixed model in which COMM opioid misuse scores were entered as a continuous independent variable. In this model, the covariance parameter of sample as random intercept was nonzero, and so the random intercept was retained to account for clustering. This model (model 2) indicated that after controlling for pain severity, MDD diagnosis, and opioid dose, higher COMM scores predicted greater anhedonia, $B=0.15, S E=0.05, p<.001$. We then computed the same model using a continuous measure of depression symptom count (current and worst episode) instead of MDD diagnosis. In this model (model 3), depression symptom count $(B=0.20, S E=$ $0.08, p=.02)$ and opioid misuse scores $(B=0.11, S E=$ $0.05, p=.03$ ) also significantly predicted anhedonia, whereas pain severity and opioid dose did not. As a final sensitivity analysis (model 4), we added opioid use duration to the set of covariates above. In this model, depression symptom count was the strongest predictor $(B=0.37, S E=0.09, p<.001)$, but opioid use duration $(B=0.01, S E=0.004, p=.036)$ and opioid misuse status $(B=2.14, S E=1.00, p=.034)$ also significantly predicted anhedonia, whereas pain severity and opioid dose did not. Also, we examined whether anhedonia levels differed by primary pain condition, but neither the omnibus $F$-test nor any of the uncorrected pairwise contrasts were significant.

\subsubsection{Sample 4}

In sample 4, anhedonia correlated significantly with depression severity as measured by BDI scores $(r$ $=0.50, p<.01)$ but not with pain severity (BPI; $r=$ $0.07, p=0.61)$.

\section{Discussion}

Here we demonstrate that individuals with chronic pain report significantly greater levels of anhedonia than a meta-analytically derived large sample of healthy controls. Moreover, across three opioid-treated chronic pain samples, anhedonic symptoms were significantly greater in patients classified as opioid misusers compared to those classified as non-misusers. The association between opioid misuse and anhedonia remained significant after controlling for individual differences in pain severity, depression, opioid dose and duration of opioid treatment. To our knowledge, this is the first report in the scientific literature to document that opioid misusers with chronic pain exhibit elevated symptoms of anhedonia. Although opioid misusers demonstrated the highest levels of anhedonia, scores in opioid users with chronic pain were not higher than anhedonia scores in a fourth chronic pain sample without 
regular opioid use, consistent with the view that anhedonia may stem from chronic pain and opioid misuse, but not from regulated use of opioid analgesics, per se.

Chronic pain has been linked to disrupted reward processing in both humans (Baliki et al., 2010, Geha et al., 2014, Loggia et al., 2013) and rodents (Schwartz et al., 2014, Thompson et al., 2018). Despite this evidence and the high comorbidity between chronic pain and depression, little data exists on the hedonic capacity of chronic pain patients. Initial findings indicated modestly increased physical anhedonia symptoms for arthritic pain patients (Marbach and Lund, 1981, Marbach et al., 1983). Here, we extend these findings and show modest, but significant increases in anhedonia scores in four diverse chronic pain samples. Though presence vs. absence of major depressive disorder diagnosis was not significantly associated with anhedonia, depression symptom severity (measured continuously) was a significant yet modest contributor to the relationship between chronic pain and anhedonia, replicating Marbach et al's results across chronic pain groups. Thus, anhedonia in chronic pain cannot be explained by comorbid depression only. Other mechanisms linking chronic pain and anhedonia include disrupted endogenous opioid signaling (Harris et al., 2007, Martikainen et al., 2013, Thompson et al., 2018), changes to mesolimbic signaling (Baliki et al., 2010, Loggia et al., 2013, Schwartz et al., Schwartz et al., 2014), prefrontal areas (Rodriguez-Raecke et al., 2009, Seminowicz et al., 2011), or the interaction between these circuits (Lee et al., 2015), and changes in attention (Crombez et al., 1996, Stefaan Van et al., 2007).

A majority of the chronic pain samples included in this study were treated with opioids. Pain conditions like neuropathic pain and fibromyalgia have been associated with reduced opioid receptor availability in the absence of opioid pharmacotherapy, however (Harris et al., 2007, Martikainen et al., 2013, Thompson et al., 2018). Accordingly, it is likely that chronic pain can cause anhedonia symptoms independently of opioid medication. Indeed, anhedonia scores were comparable between opioid treated samples and our non-user sample. Also, in the opioidtreated samples we found no significant association between opioid dose and anhedonia scores. Similarly, duration of opioid treatment, although a significant predictor, did not explain much variance in the data. Meeting criteria for opioid misuse on the other hand, was associated with significantly greater anhedonia than healthy samples and non-misusing chronic pain pa- tients. These findings are consistent with psychophysiological data from experiments involving opioidtreated chronic pain samples, showing reduced attentional and autonomic responses to rewarding stimuli in opioid misusers compared to non-misusers (Garland et al., 2017, Garland et al., 2015a).

Interestingly, the mean anhedonia scores of opioid misusing pain patients were comparable with the scores reported for patients with opioid use disorder (Garfield et al., 2017, Stevens et al., 2007, Zijlstra et $a l ., 2009$ ) and other substance use disorders (Franken et al., 2007). While anhedonia symptoms in SUD and chronic pain samples are substantially lower than those observed in patients with current MDD, the standardized effect sizes in these previous studies have been large (Garfield et al., 2017, Stevens et al., 2007, Franken et al., 2007). Chronic opioid misuse is hypothesized to increase neurobiological sensitization to aversive stimuli (i.e., stress and pain) coupled with decreased neural responsiveness to non-drug rewards (Shurman et al., 2010). This allostatic shift in reward set points is thought to result in anhedonia and a dwindling sense of subjective well-being that in turn compel dose escalation as a means of preserving hedonic equilibrium (Koob and Le Moal, 2001, Koob and Le Moal, 2008). Ironically, by virtue of the allostatic process increased consumption of opioids may lead to hyperalgesia (Arout et al., 2015), tolerance (Christie, 2008), and emotion regulation deficits (Garland et al., 2017). These changes could in turn exacerbate anhedonia and drive the downward spiral of behavioral escalation linking chronic pain to prescription opioid misuse (Garland et al., 2013).

To be clear, cross-sectional studies cannot determine whether anhedonia is the result of long-term opioid misuse and/or chronic pain. Alternatively, premorbid depression and hedonic dysfunction might increase risk for developing chronic pain and/or opioid misuse by compelling use of opioids for relief of negative affect. A recent study in college students reported significantly higher SHAPS scores in non-medical opioid users compared to non-drug using students which was unchanged at 1-year follow-up (Meshesha et al., 2017). If anhedonia is a risk mechanism undergirding the comorbidity of chronic pain opioid misuse, then interventions that aim to remediate anhedonia may be especially therapeutic for opioid misusing chronic pain patients. In that regard, a behavioral intervention that integrates mindfulness with training in savoring hedonic pleasure from natural reinforcers, MindfulnessOriented Recovery Enhancement (MORE), has decreased chronic pain severity and opioid misuse in two 
RCTs (Garland et al., 2019, Garland et al., 2014b) - effects associated with increased autonomic and neurophysiological responsiveness to natural rewards (Garland et al., 2014a, Garland et al., 2015b). It remains to be seen if MORE or other (behavioral or pharmacological) interventions can modulate subjective symptoms of anhedonia in this population.

Some methodological aspects warrant consideration. The present study compared pain samples with meta-analytically derived data from more than 2600 healthy participants tested previously in a variety of settings, countries and spanning diverse age groups. This approach is arguably better suited to generate generalizable results than the inclusion of a single control group. Importantly, the meta-analytical approach also allowed us to calculate prediction intervals (PIs) for each group. The 58 healthy samples exhibited considerable heterogeneity, as reflected in the PI, which showed overlap with the chronic pain PI. This overlap indicates that some inconsistent results can be expected in future studies comparing anhedonia in chronic pain to healthy samples. Also, the chronic pain samples included here may have differed from the healthy control samples on unmeasured psychosocial variables plausibly linked with anhedonia like socioeconomic status, marital status, and urbanity vs. rurality. Future studies should attempt to control for a broader range of potential confounders. Whilst there were systematic differences between our chronic pain samples and healthy samples in terms of age distribution, control meta-regressions showed that age and gender could not explain the reported differences in anhedonia between groups.

Study results were based on self-report of agreement with a series of hypothetical everyday rewards. Though anhedonia questionnaires tap into respondents' capacity for pleasure, responses may also be shaped by their ability to remember and/or predict pleasure. A benefit of questionnaires compared to laboratory reward tests, is the ability to assess a large range of rewards and contexts. A promising avenue for future, ecologically-valid research on anhedonia is to combine questionnaires and lab tests with experience sampling.

Ultimately, intact hedonic function is fundamental to the preservation of subjective well-being. Insofar as hedonic experience reflects optimization of internal homeostatic balance, it is sensitive to perturbations to bodily integrity. Classical philosophical accounts have posed pain in contradistinction to pleasure. Nevertheless, these two opposing phenomena are modulated by similar neurochemical processes, notably muopioids (Leknes and Tracey, 2008). Here, we show that chronic pain patients exhibit higher symptoms of anhedonia than healthy people. Reduced hedonic capacity was most pronounced in pain patients also reporting misuse of opioid medications. In sum, our data are consistent with the interpretation that both chronic pain and opioid misuse contribute to anhedonia.

\section{References}

Admon, R. \& Pizzagalli, D. A. (2015). Corticostriatal pathways contribute to the natural time course of positive mood. Nature Communications 6, 10065.

Al Aïn, S., Carré, A., Fantini-Hauwel, C., Baudouin, J.-Y. \& Besche-Richard, C. (2013). What is the emotional core of the multidimensional Machiavellian personality trait? Frontiers in Psychology 4, 454.

Ameli, R., Luckenbaugh, D. A., Gould, N. F., Holmes, M. K., Lally, N., Ballard, E. D. \& Zarate, C. A., Jr. (2014). SHAPS-C: the Snaith-Hamilton pleasure scale modified for clinician administration. PeerJ 2, e429.

Ang, Y.-S., Lockwood, P., Apps, M. A. J., Muhammed, K. \& Husain, M. (2017). Distinct Subtypes of Apathy Revealed by the Apathy Motivation Index. PLOS ONE 12, e0169938.

Arout, C. A., Edens, E., Petrakis, I. L. \& Sofuoglu, M. (2015). Targeting Opioid-Induced Hyperalgesia in Clinical Treatment: Neurobiological Considerations. CNS Drugs 29, 465-486.

Auerbach, R. P., Pisoni, A., Bondy, E., Kumar, P., Stewart, J. G., Yendiki, A. \& Pizzagalli, D. A. (2017). Neuroanatomical Prediction of Anhedonia in Adolescents. Neuropsychopharmacology 42, 2087 2095.

Baliki, M. N., Geha, P. Y., Fields, H. L. \& Apkarian, A. V. (2010). Predicting Value of Pain and Analgesia: Nucleus Accumbens Response to Noxious Stimuli Changes in the Presence of Chronic Pain. Neuron 66, 149-160.

Barch, D. M., Treadway, M. T. \& Schoen, N. (2014). Effort, anhedonia, and function in schizophrenia: Reduced effort allocation predicts amotivation and functional impairment. Journal of Abnormal Psychology 123, 387-397.

Buchel, C., Miedl, S. \& Sprenger, C. (2018). Hedonic processing in humans is mediated by an opioidergic mechanism in a mesocorticolimbic system. eLife 7, e39648.

Butler, S. F., Budman, S. H., Fernandez, K. C., Houle, B., Benoit, C., Katz, N. \& Jamison, R. N. (2007). Development and validation of the Current Opioid Misuse Measure. Pain 130, 144-56. 
Carpinelli, L., Bucci, C., Santonicola, A., Zingone, F., Ciacci, C. \& Iovino, P. (2019). Anhedonia in irritable bowel syndrome and in inflammatory bowel diseases and its relationship with abdominal pain. Neurogastroenterol Motil, e13531.

Chase, H. W., Fournier, J. C., Bertocci, M. A., Greenberg, T., Aslam, H., Stiffler, R., Lockovich, J., Graur, S., Bebko, G., Forbes, E. E. \& Phillips, M. L. (2017). A pathway linking reward circuitry, impulsive sensation-seeking and risky decision-making in young adults: identifying neural markers for new interventions. Translational Psychiatry 7, e1096.

Chelnokova, O., Laeng, B., Eikemo, M., Riegels, J., Løseth, G., Maurud, H., Willoch, F. \& Leknes, S. (2014). Rewards of Beauty: The Opioid System Mediates Social Motivation in Humans. Molecular Psychiatry 19, 746-747.

Chou, R., Fanciullo, G. J., Fine, P. G., Adler, J. A., Ballantyne, J. C., Davies, P., Donovan, M. I., Fishbain, D. A., Foley, K. M., Fudin, J. \& others (2009). Clinical guidelines for the use of chronic opioid therapy in chronic noncancer pain. The Journal of Pain 10, 113-130.

Christie, M. J. (2008). Cellular neuroadaptations to chronic opioids: tolerance, withdrawal and addiction. British Journal of Pharmacology 154, 384-396.

Chung, Y. S. \& Barch, D. (2015). Anhedonia is associated with reduced incentive cue related activation in the basal ganglia. Cognitive, Affective, \& Behavioral Neuroscience 15, 749-767.

Crombez, G., Eccleston, C., Baeyens, F. \& Eelen, P. (1996). The disruptive nature of pain: An experimental investigation. Behaviour Research and Therapy 34, 911-918.

Culbreth, A. J., Gold, J. M., Cools, R. \& Barch, D. M. (2016a). Impaired Activation in Cognitive Control Regions Predicts Reversal Learning in Schizophrenia. Schizophrenia Bulletin 42, 484-493.

Culbreth, A. J., Westbrook, A., Daw, N. D., Botvinick, M. \& Barch, D. M. (2016b). Reduced model-based decision-making in schizophrenia. Journal of Abnormal Psychology 125, 777-787.

Dean, Z., Horndasch, S., Giannopoulos, P. \& McCabe, C. (2016). Enhanced neural response to anticipation, effort and consummation of reward and aversion during bupropion treatment. Psychological Medicine 46, 2263-2274.

DerSimonian, R. \& Laird, N. (1986). Meta-analysis in clinical trials. Controlled Clinical Trials 7, 177-188. Dillon, D. G., Wiecki, T., Pechtel, P., Webb, C., Goer, F., Murray, L., Trivedi, M., Fava, M., McGrath, P. J., Weissman, M., Parsey, R., Kurian,
B., Adams, P., Carmody, T., Weyandt, S., ShoresWilson, K., Toups, M., McInnis, M., Oquendo, M. A., Cusin, C., Deldin, P., Bruder, G. \& Pizzagalli, D. A. (2015). A computational analysis of flanker interference in depression. Psychological Medicine 45, 2333-2344.

Drijgers, R. L., Verhey, F. R. J., Tissingh, G., van Domburg, P. H. M. F., Aalten, P. \& Leentjens, A. F. G. (2012). The role of the dopaminergic system in mood, motivation and cognition in Parkinson's disease: A double blind randomized placebo-controlled experimental challenge with pramipexole and methylphenidate. Journal of the Neurological Sciences 320, 121-126.

Eccleston, C. \& Crombez, G. (1999). Pain demands attention: A cognitive-affective model of the interruptive function of pain. Psychol Bull 125, 356-66. Eikemo, M., Løseth, G. E., Johnstone, T., Gjerstad, J., Willoch, F. \& Leknes, S. (2016). Sweet taste pleasantness is modulated by morphine and naltrexone. Psychopharmacology, 1-13.

Eisenstein, S. A., Bogdan, R., Chen, L., Moerlein, S. M., Black, K. J., Perlmutter, J. S., Hershey, T. \& Barch, D. M. (2017). Preliminary evidence that negative symptom severity relates to multilocus genetic profile for dopamine signaling capacity and D2 receptor binding in healthy controls and in schizophrenia. Journal of Psychiatric Research 86, 917.

Elvemo, N. A., Landrø, N. I., Borchgrevink, P. C. \& Håberg, A. K. (2015). Reward responsiveness in patients with chronic pain. European Journal of Pain, n/a-n/a.

Fervaha, G., Graff-Guerrero, A., Zakzanis, K. K., Foussias, G., Agid, O. \& Remington, G. (2013). Incentive motivation deficits in schizophrenia reflect effort computation impairments during cost-benefit decision-making. Journal of Psychiatric Research 47, 1590-1596.

Franken, I. H. A., Rassin, E. \& Muris, P. (2007). The assessment of anhedonia in clinical and non-clinical populations: Further validation of the Snaith-Hamilton Pleasure Scale (SHAPS). Journal of Affective Disorders 99, 83-89.

Gadeikis, D., Bos, N., Schweizer, S., Murphy, F. \& Dunn, B. (2017). Engaging in an experiential processing mode increases positive emotional response during recall of pleasant autobiographical memories. Behaviour Research and Therapy 92, 68-76.

Garfield, J. B. B., Cotton, S. M., Allen, N. B., Cheetham, A., Kras, M., Yücel, M. \& Lubman, D. I. (2017). Evidence that anhedonia is a symptom of 
opioid dependence associated with recent use. Drug and Alcohol Dependence 177, 29-38.

Garland, E. L., Bryan, C. J., Nakamura, Y., Froeliger, B. \& Howard, M. O. (2017). Deficits in autonomic indices of emotion regulation and reward processing associated with prescription opioid use and misuse. Psychopharmacology (Berl) 234, 621-629.

Garland, E. L., Froeliger, B. \& Howard, M. O. (2014a). Effects of Mindfulness-Oriented Recovery Enhancement on reward responsiveness and opioid cue-reactivity. Psychopharmacology 231, 3229-3238.

Garland, E. L., Froeliger, B. \& Howard, M. O. (2015b). Neurophysiological evidence for remediation of reward processing deficits in chronic pain and opioid misuse following treatment with Mindfulness-Oriented Recovery Enhancement: exploratory ERP findings from a pilot RCT. Journal of Behavioral Medicine 38, 327-336.

Garland, E. L., Froeliger, B. \& Howard, M. O. (2015a). Allostatic dysregulation of natural reward processing in prescription opioid misuse: autonomic and attentional evidence. Biol Psychol 105, 124-9.

Garland, E. L., Froeliger, B., Zeidan, F., Partin, K. \& Howard, M. O. (2013). The downward spiral of chronic pain, prescription opioid misuse, and addiction: cognitive, affective, and neuropsychopharmacologic pathways. Neurosci Biobehav Rev 37, 2597-607.

Garland, E. L., Hanley, A. W., Riquino, M. R., Reese, S. E., Baker, A. K., Salas, K., Yack, B. P., Bedford, C. E., Bryan, M. A., Atchley, R. M., Nakamura, Y., Froeliger, B. \& Howard, M. O. (2019). Mindfulness-Oriented Recovery Enhancement reduces opioid misuse risk via analgesic and positive psychological mechanisms: A randomized controlled trial. . Journal of Consulting and Clinical Psychology. Garland, E. L., Manusov, E. G., Froeliger, B., Kelly, A., Williams, J. M. \& Howard, M. O. (2014b). Mindfulness-oriented recovery enhancement for chronic pain and prescription opioid misuse: results from an early-stage randomized controlled trial. Journal of Consulting and Clinical Psychology 82, 448. Geha, P., deAraujo, I., Green, B. \& Small, D. M. (2014). Decreased food pleasure and disrupted satiety signals in chronic low back pain. PAIN® 155, 712-722. Godlewska, B. R., Masaki, C., Sharpley, A. L., Cowen, P. J. \& Emir, U. E. (2018). Brain glutamate in medication-free depressed patients: a proton MRS study at 7 Tesla. Psychological Medicine 48, 17311737.

Gradin, V. B., Pérez, A., MacFarlane, J. A., Cavin, I., Waiter, G., Engelmann, J., Dritschel, B., Pomi, A., Matthews, K. \& Steele, J. D. (2015). Abnormal brain responses to social fairness in depression: an fMRI study using the Ultimatum Game. Psychological Medicine 45, 1241-1251.

Greenberg, T., Chase, H. W., Almeida, J. R., Stiffler, R., Zevallos, C. R., Aslam, H. A., Deckersbach, T., Weyandt, S., Cooper, C., Toups, M., Carmody, T., Kurian, B., Peltier, S., Adams, P., McInnis, M. G., Oquendo, M. A., McGrath, P. J., Fava, M., Weissman, M., Parsey, R., Trivedi, M. H. \& Phillips, M. L. (2015). Moderation of the Relationship Between Reward Expectancy and Prediction Error-Related Ventral Striatal Reactivity by Anhedonia in Unmedicated Major Depressive Disorder: Findings From the EMBARC Study. American Journal of Psychiatry 172, 881-891.

Han, S. (2017). Measuring and modifying information bias in depression. University of Oxford.

Harris, R. E., Clauw, D. J., Scott, D. J., McLean, S. A., Gracely, R. H. \& Zubieta, J.-K. (2007). Decreased Central mu-Opioid Receptor Availability in Fibromyalgia. J. Neurosci. 27, 10000-10006.

Higgins, J. P. T., Thompson, S. G., Deeks, J. J. \& Altman, D. G. (2003). Measuring inconsistency in meta-analyses. BMJ 327, 557-560.

Horndasch, S., O'Keefe, S., Lamond, A., Brown, K. \& McCabe, C. (2016). Increased anticipatory but decreased consummatory brain responses to food in sisters of anorexia nervosa patients. BJPsych Open 2 , 255-261.

Huhn, A. S., Meyer, R. E., Harris, J. D., Ayaz, H., Deneke, E., Stankoski, D. M. \& Bunce, S. C. (2016). Evidence of anhedonia and differential reward processing in prefrontal cortex among post-withdrawal patients with prescription opiate dependence. Brain Research Bulletin 123, 102-109.

Huneke, N. T. M., Walsh, A. E. L., Brown, R., Browning, M. \& Harmer, C. J. (2017). No evidence for an acute placebo effect on emotional processing in healthy volunteers. Journal of Psychopharmacology 31, 1578-1587.

Kirkpatrick, M. G., Goldenson, N. I., Kapadia, N., Kahler, C. W., de Wit, H., Swift, R. M., McGeary, J. E., Sussman, S. \& Leventhal, A. M. (2016). Emotional traits predict individual differences in amphetamine-induced positive mood in healthy volunteers. Psychopharmacology 233, 89-97.

Klepstad, P., Loge, J. H., Borchgrevink, P. C., Mendoza, T. R., Cleeland, C. S. \& Kaasa, S. (2002). The Norwegian brief pain inventory questionnaire: translation and validation in cancer pain patients. $J$ Pain Symptom Manage 24, 517-25. 
Koob, G. F. \& Le Moal, M. (2001). Drug addiction, dysregulation of reward, and allostasis. Neuropsychopharmacology 24, 97-129.

Koob, G. F. \& Le Moal, M. (2008). Neurobiological mechanisms for opponent motivational processes in addiction. Philosophical Transactions of the Royal Society B: Biological Sciences 363, 3113.

Koob, G. F. \& Moal, M. L. (1997). Drug Abuse: Hedonic Homeostatic Dysregulation. Science 278, 5258 .

Kumar, P., Goer, F., Murray, L., Dillon, D. G., Beltzer, M. L., Cohen, A. L., Brooks, N. H. \& Pizzagalli, D. A. (2018). Impaired reward prediction error encoding and striatal-midbrain connectivity in depression. Neuropsychopharmacology 43, 15811588.

Kumar, P., Waiter, G., Ahearn, T., Milders, M., Reid, I. \& Steele, J. D. (2008). Abnormal temporal difference reward-learning signals in major depression. Brain 131, 2084-2093.

Lansdall, C. J., Coyle-Gilchrist, I. T. S., Jones, P. S., Vázquez Rodríguez, P., Wilcox, A., Wehmann, E., Dick, K. M., Robbins, T. W. \& Rowe, J. B. (2017). Apathy and impulsivity in frontotemporal lobar degeneration syndromes. Brain 140, 1792-1807.

Lawson, R. P., Nord, C. L., Seymour, B., Thomas, D. L., Dayan, P., Pilling, S. \& Roiser, J. P. (2017). Disrupted habenula function in major depression. Molecular Psychiatry 22, 202-208.

Lee, M., Manders, T. R., Eberle, S. E., Su, C., D'amour, J., Yang, R., Lin, H. Y., Deisseroth, K., Froemke, R. C. \& Wang, J. (2015). Activation of Corticostriatal Circuitry Relieves Chronic Neuropathic Pain. The Journal of Neuroscience 35, 5247-5259.

Leknes, S. \& Tracey, I. (2008). A common neurobiology for pain and pleasure. Nat Rev Neurosci 9, 314-320.

Liu, W.-h., Wang, L.-z., Shang, H.-r., Shen, Y., Li, Z., Cheung, E. F. C. \& Chan, R. C. K. (2014). The influence of anhedonia on feedback negativity in major depressive disorder. Neuropsychologia 53, 213-220.

Liu, W.-h., Wang, L.-Z., Zhao, S.-h., Ning, Y.-p. \& Chan, R. C. K. (2012a). Anhedonia and emotional word memory in patients with depression. Psychiatry Research 200, 361-367.

Liu, W.-h., Wang, L.-z., Zhu, Y.-h., Li, M.-h. \& Chan, R. C. K. (2012b). Clinical utility of the SnaithHamilton-Pleasure scale in the Chinese settings. BMC Psychiatry 12, 184.

Loggia, M. L., Berna, C., Kim, J., Cahalan, C. M., Gollub, R. L., Wasan, A. D., Harris, R. E., Edwards, R. R. \& Napadow, V. (2013). Disrupted brain circuitry for pain-related reward/punishment in fibromyalgia. Arthritis and Rheumatism, n/a-n/a.

Mann, C. L., Footer, O., Chung, Y. S., Driscoll, L. L. \& Barch, D. M. (2013). Spared and impaired aspects of motivated cognitive control in schizophrenia. Journal of abnormal psychology 122, 745-755.

Marbach, J. J. \& Lund, P. (1981). Depression, anhedonia and anxiety in temporomandibular joint and other facial pain syndromes. Pain 11, 73-84.

Marbach, J. J., Richlin, D. M. \& Lipton, J. A. (1983). Illness Behavior, Depression and Anhedonia in Myofascial Face and Back Pain Patients. Psychotherapy and Psychosomatics 39, 47-54.

Martikainen, I. K., Peciña, M., Love, T. M., Nuechterlein, E. B., Cummiford, C. M., Green, C. R., Harris, R. E., Stohler, C. S. \& Zubieta, J.-K. (2013). Alterations in Endogenous Opioid Functional Measures in Chronic Back Pain. The Journal of Neuroscience 33, 14729-14737.

McCabe, C., Woffindale, C., Harmer, C. J. \& Cowen, P. J. (2012). Neural Processing of Reward and Punishment in Young People at Increased Familial Risk of Depression. Biological Psychiatry 72, 588-594. Meltzer, E. C., Rybin, D., Saitz, R., Samet, J. H., Schwartz, S. L., Butler, S. F. \& Liebschutz, J. M. (2011). Identifying prescription opioid use disorder in primary care: diagnostic characteristics of the Current Opioid Misuse Measure (COMM). Pain 152, 397-402. Meshesha, L. Z., Pickover, A. M., Teeters, J. B. \& Murphy, J. G. (2017). A Longitudinal Behavioral Economic Analysis of Non-medical Prescription Opioid Use Among College Students. Psychol Rec, 241-51.

Nagashima, K., Noma, H. \& Furukawa, T. A. (2018). Prediction intervals for random-effects metaanalysis: A confidence distribution approach. Stat Methods Med Res, 962280218773520.

Narayana, A., Katz, N., Shillington, A. C., Stephenson, J. J., Harshaw, Q., Frye, C. B. \& Portenoy, R. K. (2015). National Breakthrough Pain Study: prevalence, characteristics, and associations with health outcomes. Pain 156, 252-9.

Ng, C. G., Chin, S. C., Yee, A. H. A., Loh, H. S., Sulaiman, A. H., Sherianne Sook Kuan, W. \& Habil, M. H. (2014). Validation of Malay Version of SnaithHamilton Pleasure Scale: Comparison between Depressed Patients and Healthy Subjects at an OutPatient Clinic in Malaysia. The Malaysian journal of medical sciences: MJMS 21, 62-70.

Norbury, A., Kurth-Nelson, Z., Winston, J. S., Roiser, J. P. \& Husain, M. (2015). Dopamine 
Regulates Approach-Avoidance in Human SensationSeeking. International Journal of Neuropsychopharmacology 18, pyv041.

Nugent, A. C., Ballard, E. D., Gould, T. D., Park, L. T., Moaddel, R., Brutsche, N. E. \& Zarate, C. A. (2018). Ketamine has distinct electrophysiological and behavioral effects in depressed and healthy subjects. Molecular Psychiatry.

Osuch, E. A., Manning, K., Hegele, R. A., Théberge, J., Neufeld, R., Mitchell, D., Williamson, P. \& Gardner, R. C. (2016). Depression, marijuana use and early-onset marijuana use conferred unique effects on neural connectivity and cognition. Acta Psychiatrica Scandinavica 134, 399-409.

Pechtel, P., Dutra, S. J., Goetz, E. L. \& Pizzagalli, D. A. (2013). Blunted reward responsiveness in remitted depression. Journal of Psychiatric Research 47, 18641869.

Pechtel, P. \& Pizzagalli, D. A. (2013). Disrupted reinforcement learning and maladaptive behavior in women with a history of childhood sexual abuse: A high-density event-related potential study. JAMA Psychiatry 70, 499-507.

Porcelli, A. J. \& Delgado, M. R. (2017). Stress and decision making: effects on valuation, learning, and risk-taking. Current Opinion in Behavioral Sciences 14, 33-39.

Price, R. C., Christou, N. V., Backman, S. B., Stone, L. \& Schweinhardt, P. (2016). Opioid-receptor antagonism increases pain and decreases pleasure in obese and non-obese individuals. Psychopharmacology 233, 3869-3879.

Priddy, S. E., Hanley, A. W., Riquino, M. R., Platt, K. A., Baker, A. K. \& Garland, E. L. (2018). Dispositional mindfulness and prescription opioid misuse among chronic pain patients: Craving and attention to positive information as mediating mechanisms. Drug Alcohol Depend 188, 86-93.

R Core Team (2018). R: A language and environment for statistical computing. R Foundation for Statistical Computing: Vienna, Austria.

Renfroe, J. B., Bradley, M. M., Okun, M. S. \& Bowers, D. (2016). Motivational engagement in Parkinson's disease: Preparation for motivated action. International Journal of Psychophysiology 99, 24-32.

Ricciardi, L., Ferrazzano, G., Demartini, B., Morgante, F., Erro, R., Ganos, C., Bhatia, K. P., Berardelli, A. \& Edwards, M. (2016). Know thyself: Exploring interoceptive sensitivity in Parkinson's disease. Journal of the Neurological Sciences 364, 110115 .
Rodriguez-Raecke, R., Niemeier, A., Ihle, K., Ruether, W. \& May, A. (2009). Brain Gray Matter Decrease in Chronic Pain Is the Consequence and Not the Cause of Pain. J. Neurosci. 29, 13746-13750.

Ryu, V. (2013). Dysfunctional reward learning in bipolar disorder: An event-related potential study. p. 37. Yonsei University.

Rzepa, E. \& McCabe, C. (2016). Decreased anticipated pleasure correlates with increased salience network resting state functional connectivity in adolescents with depressive symptomatology. Journal of Psychiatric Research 82, 40-47.

Scheidegger, M., Walter, M., Lehmann, M., Metzger, C., Grimm, S., Boeker, H., Boesiger, P., Henning, A. \& Seifritz, E. (2012). Ketamine Decreases Resting State Functional Network Connectivity in Healthy Subjects: Implications for Antidepressant Drug Action. PLOS ONE 7, e44799.

Schwartz, N., Miller, C. \& Fields, H. L. CorticoAccumbens Regulation of Approach-Avoidance Behavior Is Modified by Experience and Chronic Pain. Cell Reports 19, 1522-1531.

Schwartz, N., Temkin, P., Jurado, S., Lim, B. K., Heifets, B. D., Polepalli, J. S. \& Malenka, R. C. (2014). Decreased motivation during chronic pain requires long-term depression in the nucleus accumbens. Science 345, 535-542.

Seminowicz, D. A., Wideman, T. H., Naso, L., Hatami-Khoroushahi, Z., Fallatah, S., Ware, M. A., Jarzem, P., Bushnell, M. C., Shir, Y., Ouellet, J. A. \& Stone, L. S. (2011). Effective Treatment of Chronic Low Back Pain in Humans Reverses Abnormal Brain Anatomy and Function. The Journal of Neuroscience 31, 7540-7550.

Sheehan, D. V., Lecrubier, Y., Sheehan, K. H., Amorim, P., Janavs, J., Weiller, E., Hergueta, T., Baker, R. \& Dunbar, G. C. (1998). The MiniInternational Neuropsychiatric Interview (M.I.N.I.): the development and validation of a structured diagnostic psychiatric interview for DSM-IV and ICD10. J Clin Psychiatry 59 Suppl 20, 22-33; quiz 34-57.

Shurman, J., Koob, G. F. \& Gutstein, H. B. (2010). Opioids, pain, the brain, and hyperkatifeia: a framework for the rational use of opioids for pain. Pain medicine (Malden, Mass.) 11, 1092-1098.

Snaith, R., Hamilton, M., Morley, S., Humayan, A., Hargreaves, D. \& Trigwell, P. (1995). A scale for the assessment of hedonic tone the Snaith-Hamilton Pleasure Scale. Br J Psychiatry 167, 99-103.

Sprengelmeyer, R., Steele, J. D., Mwangi, B., Kumar, P., Christmas, D., Milders, M. \& Matthews, K. (2011). The insular cortex and the neuroanatomy of 
major depression. Journal of Affective Disorders 133, 120-127.

Sprenger, T., Valet, M., Boecker, H., Henriksen, G., Spilker, M., Willoch, F., Wagner, K., Wester, H. \& Tolle, T. (2006). Opioidergic activation in the medial pain system after heat pain. Pain 122, 63-7.

Steele, J. D., Kumar, P. \& Ebmeier, K. P. (2007). Blunted response to feedback information in depressive illness. Brain 130, 2367-2374.

Stefaan Van, D., Geert, C. \& Jürgen, L. (2007). Pain Draws Visual Attention to Its Location: Experimental Evidence for a Threat-Related Bias. The Journal of Pain 8, 976-982.

Stevens, A., Peschk, I. \& Schwarz, J. (2007). Implicit learning, executive function and hedonic activity in chronic polydrug abusers, currently abstinent polydrug abusers and controls. Addiction 102, 937-946.

Thomas, E. A. \& Garland, E. L. (2017). Mindfulness is Associated With Increased Hedonic Capacity Among Chronic Pain Patients Receiving Extended Opioid Pharmacotherapy. Clin J Pain 33, 166-173.

Thompson, S. J., Pitcher, M. H., Stone, L. S., Tarum, F., Niu, G., Chen, X., Kiesewetter, D. O., Schweinhardt, P. \& Bushnell, M. C. (2018). Chronic neuropathic pain reduces opioid receptor availability with associated anhedonia in rat. 159, 1856-1866.

Tudge, L., Williams, C., Cowen, P. J. \& McCabe, C. (2015). Neural Effects of Cannabinoid CB1 Neutral Antagonist Tetrahydrocannabivarin on Food Reward and Aversion in Healthy Volunteers. International Journal of Neuropsychopharmacology 18, pyu094.

Ubl, B., Kuehner, C., Kirsch, P., Ruttorf, M., Diener, C. \& Flor, H. (2015). Altered neural reward and loss processing and prediction error signalling in depression. Social Cognitive and Affective Neuroscience 10, 1102-1112.

Veroniki, A. A., Jackson, D., Viechtbauer, W., Bender, R., Bowden, J., Knapp, G., Kuss, O., Higgins, J. P., Langan, D. \& Salanti, G. (2016). Methods to estimate the between-study variance and its uncertainty in meta-analysis. Research Synthesis Methods 7, 55-79.

Viechtbauer, W. (2010). Conducting Meta-Analyses in $\mathrm{R}$ with the metafor Package. Journal of Statistical Software 36, 1-48.

Volkow, N. D. \& McLellan, A. T. (2016). Opioid Abuse in Chronic Pain--Misconceptions and Mitigation Strategies. N Engl J Med 374, 1253-63.

Vowles, K. E., McEntee, M. L., Julnes, P. S., Frohe, T., Ney, J. P. \& van der Goes, D. N. (2015). Rates of opioid misuse, abuse, and addiction in chronic pain: a systematic review and data synthesis. Pain 156, 56976.

Vrieze, E., Ceccarini, J., Pizzagalli, D. A., Bormans, G., Vandenbulcke, M., Demyttenaere, K., Van Laere, K. \& Claes, S. (2013). Measuring extrastriatal dopamine release during a reward learning task. Human Brain Mapping 34, 575-586.

Walsh, A. E., Browning, M., Drevets, W. C., Furey, M. \& Harmer, C. J. (2018). Dissociable temporal effects of bupropion on behavioural measures of emotional and reward processing in depression. Philosophical Transactions of the Royal Society B 373, 20170030.

Yoshida, K., Shimizu, Y., Yoshimoto, J., Takamura, M., Okada, G., Okamoto, Y., Yamawaki, S. \& Doya, K. (2017). Prediction of clinical depression scores and detection of changes in whole-brain using resting-state functional MRI data with partial least squares regression. PLOS ONE 12, e0179638.

Young, K. D., Bellgowan, P. S. F., Bodurka, J. \& Drevets, W. C. (2013). Behavioral and neurophysiological correlates of autobiographical memory deficits in patients with depression and individuals at high risk for depression. JAMA Psychiatry 70, 698-708.

Young, K. D., Bellgowan, P. S. F., Bodurka, J. \& Drevets, W. C. (2014). Neurophysiological correlates of autobiographical memory deficits in currently and formerly depressed subjects. Psychological Medicine 44, 2951-2963.

Zijlstra, F., Veltman, D. J., Booij, J., van den Brink, W. \& Franken, I. H. (2009). Neurobiological substrates of cue-elicited craving and anhedonia in recently abstinent opioid-dependent males. Drug Alcohol Depend 99, 183-92.

Zubieta, J.-K., Smith, Y. R., Bueller, J. A., Xu, Y., Kilbourn, M. R., Jewett, D. M., Meyer, C. R., Koeppe, R. A. \& Stohler, C. S. (2001). Regional mu opioid receptor regulation of sensory and affective dimensions of pain. Science 293, 311-315. 